\title{
Detection of early renal injury in children with solid tumors undergoing chemotherapy by urinary neutrophil gelatinase-associated lipocalin
}

\author{
MOHAMED A. ALMALKY ${ }^{1}$, SHERIEFA A. HASAN ${ }^{1}$, TAMER H. HASSAN $^{1}$, DOAA A. SHAHBAH ${ }^{1}$, \\ MOHAMED A. ARAFA ${ }^{1}$, NAGLAAA. KHALIFA ${ }^{2}$ and RASHA E. IBRAHIM ${ }^{1}$ \\ Departments of ${ }^{1}$ Pediatrics and ${ }^{2}$ Clinical Pathology, Faculty of Medicine, Zagazig University, Zagazig 44111, Egypt
}

Received December 11, 2014; Accepted January 14, 2015

DOI: $10.3892 / \mathrm{mco} .2015 .631$

\begin{abstract}
Acute kidney injury (AKI) is a complication in children with solid tumors undergoing chemotherapy, as it may prevent the use of therapy protocols and also hinder the supportive and diagnostic procedures. Thus, there is an urgent requirement for early predictive biomarkers of AKI. The most promising novel AKI biomarker is neutrophil gelatinase-associated lipocalin (NGAL). The aim of the present study was to compare the predictability of NGAL as a biomarker of AKI with creatinine as a traditional biomarker in children with solid tumors under chemotherapy. The study was performed on 30 patients with different types of solid tumors (reuroblastoma, Wilms tumor, medulloblastoma, rhabdomyosarcoma and Ewing sarcoma) and 20 control subjects. Urinary NGAL (uNGAL) and serum creatinine samples were taken three times: Baseline before the beginning of the treatment, one week after chemotherapy and at the end of the chemotherapy protocol. AKI is defined as a change in creatinine level by $>50 \%$ of the baseline. The creatinine level only rises to this level in the third sample, while uNGAL increases significantly in the second and third samples with percentage of change 376.8 and $698.2 \%$, respectively, which is highly significant $(\mathrm{P}<0.001)$. When comparing the predictive value of serum creatinine for AKI depending on the receiver operating characteristic curve with that of $\mathrm{UNGAL}$, the area under the curve (AUC) for creatinine was 0.60 with a standard error (SE) of 0.086 and $95 \%$ confidence interval $(\mathrm{CI})$ between 0.432 and 0.768 , while that of UNGAL was highly predictive with an AUC of 0.847 , SE 0.55 and $95 \%$ CI between 0.739 and 0.955 . Depending only on the creatinine level for detecting the AKI will markedly delay the diagnosis; however, uNGAL is detected earlier, and
\end{abstract}

Correspondence to: Dr Tamer H. Hassan, Department of Pediatrics, Zagazig University, No. 1 Zagazig University Street, Zagazig 44111, Egypt

E-mail: dr.tamerhassan@yahoo.com

Key words: neutrophil gelatinase-associated lipocalin, acute renal injury, children, solid tumors, chemotherapy is easier and more reliable as a marker for AKI in children with solid tumors undergoing chemotherapy.

\section{Introduction}

Acute kidney injury (AKI) is defined as an absolute increase in serum creatinine level by either $>0.3 \mathrm{mg} / \mathrm{dl}$, an increase of $\geq 50 \%$ from baseline, a reduction of the glomerular filtration rate $(\mathrm{GFR}) \geq 25 \%$ from the baseline or a reduction of the urine output by $<0.5 \mathrm{ml} / \mathrm{kg}$ for $>8 \mathrm{~h}$ (1). The requirement to describe AKI precisely and sensitively has caused the development of a multidimensional AKI classification system, proposed by the Acute Dialysis Quality Initiative group and expanded by the Acute Kidney Injury Network that brought the Risk, Injury, Failure, Loss and End-Stage renal disease (RIFLE) criteria and staging into position as the standard definition and diagnosis of this syndrome $(2,3)$. The advantages of using RIFLE criteria are that a diagnosis can be established at the stage of preventable renal dysfunction. The etiology of AKI over the past decades has shifted from primary renal disease to multifactorial causes, particularly in hospitalized children (4).

Solid tumors represent $>40 \%$ of all childhood malignancies. Examples of solid tumors are neuroblastoma, Wilms tumor, hepatoblastoma, rhabdomyosarcoma, brain tumors and sarcomas (5). Renal complications in children with malignancies primarily arise from renal parenchymal tumors, tumor lysis syndrome, malignant infiltration or obstruction of the urinary tract, or it may be secondary to a variety of treatment modalities, such as chemotherapy, surgery or radiotherapy; and finally, it can result from supportive measures (6). Chemotherapy can cause nephrotoxicity, while renal impairment can result in altered excretion and metabolism of chemotherapeutic agents. Renal dysfunction is a problematic adverse effect that can hinder the continued administration of anticancer treatment and the optimal use of ancillary and supportive measures (7).

There is an urgent requirement for early predictive biomarkers of AKI, as early intervention can significantly improve the prognosis. AKI is largely asymptomatic, and establishing the diagnosis in this increasingly common disorder currently depends on functional biomarkers, such as serial serum creatinine measurements. However, serum creatinine 
is a delayed and unreliable indicator of AKI due to various reasons (8). Neutrophil gelatinase-associated lipocalin (NGAL) is emerging as a biomarker in the urine and plasma, for the early prediction of AKI and for the prognosis of AKI in several common clinical scenarios (9). These include cardiopulmonary bypass $(10)$, kidney transplantation $(11,12)$, diarrhea-associated hemolytic uremic syndrome (13), contrast nephropathy (14) and Henoch-Schonlein purpura nephritis (15). However, the expression of NGAL in the urine of children administered chemotherapy for solid tumors has yet to be elucidated.

The aim of the present study was to compare the predictability of urinary NGAL (uNGAL) as a biomarker of AKI with creatinine as a traditional biomarker in children with solid tumors under chemotherapy.

\section{Patients and methods}

Subjects. The cross sectional study was conducted in the Pediatric Oncology Unit, Zagazig University Hospital (Zagazig, Egypt) during the period between March 2011 and March 2013. The patient group included 30 male and female patients, aged from one month to 18 years, with different solid tumors diagnosed at the Pediatric Oncology Unit, Zagazig University Hospital using the routine methods of diagnosis of solid tumors (history, clinical examination and investigations including biopsy and radiology). There was no renal impairment at the beginning of the study, as guided by the serum creatinine level. All the patients completed their protocol of therapy. The control group included 20 age- and gender-matched controls with no renal impairment, as guided by history, clinical examination and serum creatinine.

Methods. All the participants in the study were subjected to a full history and thorough clinical examination. Kidney function tests, including serum creatinine and blood urea nitrogen (BUN), were performed three times. The first sample was the baseline before the beginning of the protocol. The second sample was taken one week after the beginning of chemotherapy. The third sample was obtained at the end of the treatment protocol. Serum creatinine and BUN were measured using a Dimension RxL autoanalyzer (Siemens Healthcare Diagnostics Inc., Newark, DE, USA). The uNGAL ELISA assay was analyzed at the same time points with serum creatinine.

Sampling and principle of the $u N G A L$ assay. Morning urine samples were aseptically collected and voided directly into a sterile container. The samples were centrifuged to remove particulate matter, aliquoted and stored at $-20^{\circ} \mathrm{C}$ until the measurement using the commercial uNGAL ELISA kit (Quantikine; R\&D Systems, Minneapolis, MN, USA) in accordance with the manufacturer's instructions. The UNGAL cut-off level of $25 \mathrm{ng} / \mathrm{ml}$ was used for the definition of AKI, as based on the studies by Cho et al (16) and de Geus et al (17).

Statistical analysis. Statistical analyses were performed using SPSS 10.0 statistical software (SPSS, Inc., Chicago, IL, USA). Data are presented as the mean \pm standard deviation for quantitative variables and as numbers and percentages for
Table I. Age and gender data of cases and controls.

\begin{tabular}{lcccc}
\hline Characteristics & Cases & Controls & $\begin{array}{c}\text { t-test } \\
\text { value }\end{array}$ & P-value \\
\hline Gender, n (\%) & & & & \\
$\quad$ Males & $15(50)$ & $13(65)$ & & \\
Females & $15(50)$ & $7(35)$ & $\chi^{2}=1.1$ & 0.360 \\
Total & $30(100)$ & $20(100)$ & & \\
Age, years & $4.9 \pm 3.1$ & $6.6 \pm 3.1$ & $\mathrm{t}=1.85$ & 0.183 \\
\hline
\end{tabular}

Age is mean \pm standard deviation.

qualitative variables. The independent t-test value, $\chi^{2}$ test and correlation coefficient were used when appropriate. $\mathrm{P}<0.05$ was considered to indicate a statistically significant difference. The performance characteristics of the UNGAL levels and serum creatinine in detecting the acute renal injury were described using the area under a receiver operator characteristic (ROC) curve. Confidence intervals (CI) refer to $95 \%$ boundaries.

Ethics. The study was conducted in accordance with the Helsinki Declaration 1964 as revised in 2000 (18), and was approved by the Ethics Committee of the Faculty of Medicine, Zagazig University. Informed consent was obtained from all the study parents or guardians.

\section{Results}

Patient characteristics and changes between the three samples. The patients were $50 \%$ female and $50 \%$ male, and the mean age was $4.9 \pm 3.1$ years. The age and gender data of the studied groups are represented in Table I. There were 12 cases with Wilm's tumor (40.0\%), 10 with neuroblastoma $(33.3 \%)$, four with medulloblastoma (13.3\%), three with rhabdomyosarcoma (10.0\%), and one case of Ewing sarcoma $(3.3 \%)$. There was no significant difference in serum creatinine level between cases and controls in the baseline samples. The mean of serum creatinine was $0.4 \pm 0.1$ in patients vs. $0.36 \pm 0.13 \mathrm{mg} / \mathrm{dl}$ in controls $(\mathrm{P}>0.05)$. However, there was a highly significant difference in the serum creatinine level between the cases and controls in the second and third samples, where the means of serum creatinine in cases were $0.5 \pm 0.1$ and $0.6 \pm 0.12 \mathrm{mg} / \mathrm{dl}$, respectively vs. $0.36 \pm 0.13$ and $0.4 \pm 0.15 \mathrm{mg} / \mathrm{dl}$ in controls $(\mathrm{P}<0.001)$. The percentage of change in mean creatinine in patients in the second and third results compared to the baseline was 26.1 and $54.1 \%$, respectively. Although these two percentages were statistically significant $(\mathrm{P}<0.003$ and $\mathrm{P}<0.001$, respectively), a percentage of $26.1 \%$ in the second sample is not enough to diagnose AKI in these patients. Regarding uNGAL, there was a significant difference between cases and controls in the initial baseline results, in the second and third samples following chemotherapy initiation and at the end of the protocol, respectively. The mean of uNGAL in the first, second and third samples in order was $7.5 \pm 4.2,31.3 \pm 17.3$ and $49 \pm 16.2 \mathrm{ng} / \mathrm{ml}$ in cases vs. $3.6 \pm 1.2,3.2 \pm 1.3$ and $3.5 \pm 1.6 \mathrm{ng} / \mathrm{ml}$ in controls $(\mathrm{P}<0.001)$. The percentage of change in mean $\mathrm{UNGAL}$ in the second and 
Table II. Serum creatinine and uNGAL values in cases and controls in the three samples.

\begin{tabular}{|c|c|c|c|c|}
\hline Time points & Cases $(n=30)$ & Controls $(n=20)$ & t-test value & P-value \\
\hline \multicolumn{5}{|l|}{ First sample } \\
\hline S. creatinine, $\mathrm{mg} / \mathrm{dl}$ (range) & $0.41 \pm 0.1(0.3-0.6)$ & $0.35 \pm 0.1(0.2-0.5)$ & 1.24 & 0.168 \\
\hline uNGAL, ng/ml (range) & $7.5 \pm 4.2(4-22)$ & $3.6 \pm 1.2(1.1-6.2)$ & 3.147 & $<0.003$ \\
\hline \multicolumn{5}{|l|}{ Second sample } \\
\hline S. creatinine, mg/dl (range) & $0.5 \pm 0.1(0.3-0.8)$ & $0.36 \pm 0.13(0.18-0.45)$ & 3.58 & $<0.001$ \\
\hline uNGAL, ng/ml (range) & $31.3 \pm 17.3(10-70)$ & $3.2 \pm 1.3(1.4-5.2)$ & 5.122 & $<0.001$ \\
\hline \multicolumn{5}{|l|}{ Third sample } \\
\hline S. creatinine, $\mathrm{mg} / \mathrm{dl}$ (range) & $0.6 \pm 0.12(0.5-0.8)$ & $0.4 \pm 0.15(0.2-0.7)$ & 5.4 & $<0.001$ \\
\hline uNGAL, ng/ml (range) & $49.0 \pm 16.2(16-82)$ & $3.5 \pm 1.6(1.3-7)$ & 12.6 & $<0.001$ \\
\hline
\end{tabular}

Data are mean \pm standard deviation. First sample, baseline; second, one week after the initiation of chemotherapy; third; end of chemotherapy protocol. S. creatinine, serum creatinine; uNGAL, urinary neutrophil gelatinase-associated lipocalin.

Table III. Serum creatinine level in patients and the percentages of change in the three samples.

\begin{tabular}{lcccr}
\hline Time points & Mean + SD & Range & Change, $\%$ & Paired t-test \\
\hline First sample & $0.4 \pm 0.1$ & $0.3-0.6$ & & \\
Second sample & $0.5 \pm 0.1$ & $0.3-0.8$ & 26.1 & 3.95 \\
Third sample & $0.6 \pm 0.1$ & $0.5-0.8$ & 54.1 & 2.73 \\
\hline
\end{tabular}

First sample, baseline; second, one week after the initiation of chemotherapy; third; end of chemotherapy protocol. SD, standard deviation.

Table IV. uNGAL in patients and the percentages of change in the three samples.

\begin{tabular}{lccrr}
\hline Time points & Mean \pm SD & Range & Change, $\%$ & Paired t-test \\
\hline First sample & $7.5 \pm 4.2$ & $4-22$ & & \\
Second sample & $31.3 \pm 17.3$ & $10-70$ & 376.8 & 8.13 \\
Third sample & $49.0 \pm 16.2$ & $16-82$ & 698.2 & 14.40 \\
\hline
\end{tabular}

First sample, baseline; second, one week after the initiation of chemotherapy; third; end of chemotherapy protocol. uNGAL, urinary neutrophil gelatinase-associated; SD, standard deviation.

third samples compared to the baseline was 376.8 and $698.2 \%$, respectively, which is highly significant $(\mathrm{P}<0.001)$. Table II illustrates serum creatinine and UNGAL values in cases and controls in the three samples. The percentage of change in serum creatinine and UNGAL in the three samples of the cases is illustrated in Tables III and IV, respectively, and the correlation between the two is represented in Table V. There was a highly statistically significant difference between the percentage of patients diagnosed with AKI based on UNGAL and serum creatinine in the second sample (one week after chemotherapy initiation). AKI cases based on UNGAL were 10 out of $30(33.3 \%)$ vs. 0 out of $30(0.0 \%)$ when based on serum creatinine $(\mathrm{P}=0.002)$. However, in the third sample (after the end of the chemotherapy protocol), there was no significant difference between the percentage of patients with AKI based on UNGAL and serum creatinine. AKI cases based on UNGAL were 14 out of $30(46.7 \%)$ vs. 12 out of $30(40.0 \%)$ when based
Table V. Correlation between uNGAL and serum creatinine levels in patients.

\begin{tabular}{lcc}
\hline Variables & Correlation coefficient, $\mathrm{r}$ & P-value \\
\hline First sample & $>0.05$ & 0.30 \\
Second sample & $<0.001$ & 0.33 \\
Third sample & $<0.001$ & 0.63 \\
\hline
\end{tabular}

First sample, baseline; second, one week after the initiation of chemotherapy; third; end of chemotherapy protocol. uNGAL, urinary neutrophil gelatinase-associated lipocalin.

on serum creatinine $(\mathrm{P}=0.068)$. Table VI illustrates the AKI cases based on uNGAL and serum creatinine in patients. When comparing the predictive value of serum creatinine for 
Table VI. AKI in patients based on uNGAL and serum creatinine.

\begin{tabular}{|c|c|c|c|c|}
\hline Time points & AKI based on uNGAL & AKI based on S. creatinine & $\chi^{2}$ value & P-value \\
\hline First sample & $0(0.0 \%)$ & $0(0.0 \%)$ & & \\
\hline Second sample & $10(33.3 \%)$ & $0(0.0 \%)$ & 9.720 & 0.002 \\
\hline Third sample & $14(46.7 \%)$ & $12(40.0 \%)$ & 0.795 & 0.0680 \\
\hline
\end{tabular}

First sample, baseline; second, one week after the initiation of chemotherapy; third; end of chemotherapy protocol. AKI, acute kidney injury; S. creatinine, serum creatinine; uNGAL, urinary neutrophil gelatinase-associated lipocalin; AKI based on uNGAL by uNGAL level > $25 \mathrm{ng} / \mathrm{ml}$; AKI based on serum creatinine by an increase of $>50 \%$ from baseline.

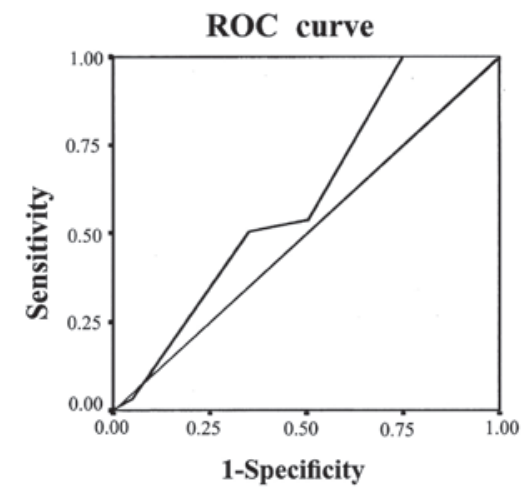

Diagonal segments are produced by ties

Figure 1. Receiver operating characteristic (ROC) curve for serum creatinine. ROC curve of serum creatinine shows that the area under the curve was 0.60 , with a standard error of 0.086 and $95 \%$ confidence interval between 0.432 and 0.768 .

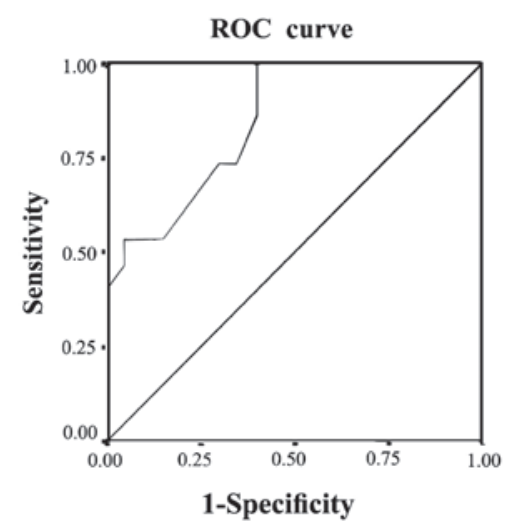

Diagonal segments are produced by ties

Figure 2. Receiver operating characteristic (ROC) curve for urinary neutrophil gelatinase-associated lipocalin (uNGAL). ROC curve of UNGAL shows that the area under the curve was 0.847 , with a standard error of 0.55 and $95 \%$ confidence interval between 0.739 and 0.955 .

AKI depending on the ROC curve with that of uNGAL, the area under the curve (AUC) for creatinine was 0.60 with a standard error (SE) of 0.086 and $95 \%$ confidence interval (CI) between 0.432 and 0.768 , while that of uNGAL was highly predictive with AUC 0.847, SE 0.55 and $95 \%$ CI between 0.739 and 0.955 . The ROC curves of serum creatinine and uNGAL are illustrated in Figs 1 and 2, respectively.

\section{Discussion}

In total, $5-10 \%$ of all hospitalized patients and $<40 \%$ of critically ill patients are estimated to experience an episode of AKI during the course of their illness $(19,20)$. The AKI complications include increased susceptibility to infections, extra-renal organ damage, chronic kidney disease and an increased rate of hospital readmission $(21,22)$.

Acute renal failure (ARF) is a serious complication of malignancies that causes substantial morbidity and mortality. Among critically ill cancer patients (CICPs), 12-49\% experience ARF and 9-32\% require renal replacement therapy during their time at an intensive care unit (ICU) (23-27). The ARF risk appears to be higher in CICPs compared to other critically ill patients $(24,28)$. In CICPs, acute renal dysfunction usually occurs in the context of multiple organ dysfunctions and is associated with mortality rates ranging from $72-85 \%$ when renal replacement therapy is required $(23,24)$.

NGAL was identified by microarray analysis as one of the earliest and strongly-induced proteins in the kidney following ischemic or nephrotoxic injury in animals and humans, where it was easily detected in the blood and urine soon after AKI (29).

Serum and UNGAL have been heavily studied in different clinical scenarios for early prediction and prognosis of AKI (9). However, to the best of our knowledge there are no studies concerning the role of NGAL as an AKI marker in children with solid tumors undergoing chemotherapy.

In the present study, there was no significant difference in the serum creatinine between cases and controls in baseline samples; however, there was a highly significant difference between the two groups one week after the initiation of therapy and at the end of the chemotherapy protocol. The percentage of change in the creatinine level at the second and third time-points was 26.1 and $54.1 \%$; however, although highly significant in comparison to the baseline, a $26.1 \%$ rise in serum creatinine is not sufficient for diagnosis of AKI according to the finding published by Mehta et al (30), which stated that AKI is defined as a change in creatinine level by $>50 \%$ of the baseline. Therefore, it is clear that the serum creatinine level in the present study only rises $>50 \%$ in the third sample, meaning that depending only on the creatinine level for adequately detecting the AKI markedly delays the diagnosis. This result is in agreement with de Geus et al (17) and Waikar et al (31) who confirmed the poor predictive value of creatinine for AKI detection, particularly in the early stage of renal injury. 
Regarding uNGAL, there was a significant difference between cases and controls in the first baseline results, in the second and third samples after chemotherapy initiation and at the end of the protocol. The percentage of change was 376.8 and $698 \%$, respectively, which is highly significant.

There was a highly statistically significant difference between the percentage of patients diagnosed with AKI based on UNGAL and serum creatinine in the second sample one week after chemotherapy initiation. AKI cases based on uNGAL were 10 out of $30(33.3 \%)$ vs. 0 out of $30(0.0 \%)$ when based on serum creatinine $(\mathrm{P}=0.002)$. However, in the third sample after the end of the chemotherapy protocol, there was no significant difference between the percentage of patients with AKI based on UNGAL and serum creatinine. AKI cases based on uNGAL were 14 out of $30(46.7 \%)$ vs. 12 out of 30 $(40.0 \%)$ when based on serum creatinine $(\mathrm{P}=0.068)$. These data are confirmed when comparing the predictive value of serum creatinine for AKI detection depending on the ROC curve with that of UNGAL, which showed that UNGAL is a good predictor of AKI with an AUC of 0.847. These data agree with those published by Haase et al (32), which is, to the best of our knowledge, the only published meta-analysis for the accuracy of uNGAL as a predictive parameter of AKI. The data also agree with Haase-Fielitz et al (33) who strongly supported the use of NGAL as a predictor of AKI, as well as subsequent initiation of renal replacement therapy with high sensitivity and specificity. Numerous studies have tested the probability of UNGAL as a predictor of AKI in pediatrics, such as the study conducted by Mishra et al (34) who studied 71 children undergoing cardiac surgery and identified AKI (RIFLE stage R or worse) in 20 patients. The study concluded that NGAL (with a cut-off value, $>50 \mathrm{ng} / \mathrm{ml}$ ) is an early predictor of AKI with an AUC of $0.99,100 \%$ sensitivity and $98 \%$ specificity. Parikh et al (35) also conducted a study on 311 children undergoing cardiac surgery and identified AKI (renal replacement therapy or doubling in creatinine) in 53 patients, and concluded that uNGAL (with a cut-off value, $>70 \mathrm{ng} / \mathrm{ml}$ ) is an early predictor of AKI with an AUC of 0.71 , $42 \%$ sensitivity and $85 \%$ specificity. UNGAL has also been investigated by Zappitelli et al (36) who studied 140 critically ill patients; 106 developed AKI and it was identified that uNGAL (cut-off value $>1.5 \mu \mathrm{g} / \mathrm{mg}$ urine creatinine) was a predictor of AKI with an AUC of $0.78,54 \%$ sensitivity and $97 \%$ specificity. Du et al (37) performed a study on 250 critically ill children, in which 18 developed AKI (pediatric RIFLE), and UNGAL was identified as a predictor of AKI with an AUC of 0.8. Wheeler et al (38) studied serum NGAL as a marker of AKI in intensive care children and had comparable results. According to de Geus et al (17), NGAL measured at ICU admission predicted the development of severe AKI similarly to serum-creatinine-derived estimated GFR (eGFR). However, when patients with an eGFR $<60 \mathrm{ml} / \mathrm{min} / 1.73 \mathrm{~m}^{2}$ at ICU admission were excluded, plasma (AUC 0.75) and urine NGAL (AUC 0.79) exhibited improved diagnostics over serum creatinine (AUC 0.65) and eGFR (AUC 0.67) for predicting AKI. Martensson et al (39) studied the impact of inflammation on NGAL concentrations in plasma and urine in patients with systemic inflammatory response syndrome, severe sepsis and septic shock with and without AKI, and concluded that plasma (AUC 0.85) and urine NGAL (AUC 0.86) were good predictors of AKI developing within the subsequent $12 \mathrm{~h}$. However, the ability of plasma NGAL to predict AKI in patients with septic shock was less powerful (AUC 0.67) compared to urine NGAL (AUC 0.86). In a recent multi-centre study investigating five urinary biomarkers in 1,635 emergency department patients at the time of hospital admission, NGAL was the most useful biomarker ( $81 \%$ specificity, $68 \%$ sensitivity at a $104 \mathrm{ng} / \mathrm{ml}$ cut-off) and predictor of the severity and duration of AKI (40). Rahimzadeh et al (41) studied 27 children post-transplant, and measured NGAL 6-12 h after kidney transplantation (cut-off value, $>175 \mathrm{ng} / \mathrm{ml}$ ), which showed an overall superior performance (AUC 0.95, 100\% sensitivity and $95.5 \%$ specificity) for the prediction of delayed graft rejection.

By contrast, Royakkers et al (42) investigated systemic NGAL and uNGAL in 140 critically ill adults, defined with AKI by RIFLE stage R or worse, and concluded that systemic NGAL and UNGAL are poor predictors of AKI in unselected critically ill patients with an AUC of 0.45 for systemic NGAL and 0.45 for UNGAL. In the study by Endre et al (43), among the general adult ICU patients, 82 subjects developed AKI within $48 \mathrm{~h}$ of admission and the predictive performance for NGAL, corrected for urinary creatinine concentration, yielded an AUC of 0.55. Metzger et al (44) compared the classification performance of urinary proteome analysis with classical markers. For uNGAL, the ROC analysis revealed a low classification accuracy with an AUC of 0.54.

Regardless of the large number of studies concerning the use of NGAL as a predictor of AKI in different situations, except for the study by Mishra et al (29) on a murine model for cisplatin nephrotoxicity which revealed that NGAL represents an early and quantitative urinary biomarker for cisplatin nephrotoxicity when compared to serum creatinine and urinary $\mathrm{N}$-acetyl- $\beta$-D-glucosaminidase, the present study is the first to evaluate NGAL in patients with solid tumors. However, numerous studies have been performed to assess renal toxicity in Wilm's tumor, such as Daw et al (45) and Bailey et al (46), but these depend on other parameters not including uNGAL.

In conclusion, uNGAL is a novel marker for the detection of early kidney injury in children with solid tumors under chemotherapy. Larger studies are required to support the conclusions drawn from the present study.

\section{References}

1. Akcan-Arikan A, Zappitelli M, Loftis LL, Washburn KK, Jefferson LS and Goldstein SL: Modified RIFLE criteria in critically ill children with acute kidney injury. Kidney Int 71: 1028-1035, 2007.

2. Srisawat N, Hoste EE and Kellum JA: Modern classification of acute kidney injury. Blood Purif 29: 300-307, 2010.

3. Adiyanti SS and Loho T: Acute Kidney Injury (AKI) biomarker. Acta Med Indones 44: 246-255, 2012.

4. Andreoli SP: Acute kidney injury in children. Pediatr Nephrol 24: 253-263, 2009.

5. Memon F, Rathi SL and Memon MH: Pattern of solid paediatric malignant neoplasm at Lumhs, Jamshoro, Pakistan. J Ayub Med Coll Abbottabad 19: 55-57, 2007.

6. Launay-Vacher V, Izzedine H, Rey JB, Rixe O, Chapalain S, Nourdine S, Paci A, Bourget P and Deray G: Incidence of renal insufficiency in cancer patients and evaluation of information available on the use of anticancer drugs in renally impaired patients. Med Sci Monit 10: CR209-CR212, 2004.

7. Kintzel PE: Anticancer drug-induced kidney disorders. Drug Saf 24: 19-38, 2001. 
8. Murray PT, Devarajan P, Levey AS, Eckardt KU, Bonventre JV, Lombardi R, Herget-Rosenthal S and Levin A: A framework and key research questions in AKI diagnosis and staging in different environments. Clin J Am Soc Nephrol 3: 864-868, 2008.

9. Devarajan P: Biomarkers for the early detection of acute kidney injury. Curr Opin Pediatr 23: 194-200, 2011.

10. Dent CL, Ma Q, Dastrala S, Bennett M, Mitsnefes MM Barasch J and Devarajan P: Plasma NGAL predicts acute kidney injury, morbidity and mortality after pediatric cardiac surgery: A prospective uncontrolled cohort study. Crit Care 11: R127, 2007.

11. Mishra J, Ma Q, Kelly C, Mitsnefes M, Mori K, Barasch J and Devarajan P: Kidney NGAL is a novel marker of acute injury following transplantation. Pediatr Nephrol 21: 856-863, 2006.

12. Parikh CR, Jani A, Mishra J, Ma Q, Kelly C, Barasch J, Edelstein CL and Devarajan P: Urine NGAL and IL-18 are predictive biomarkers for delayed graft function following kidney transplantation. Am J Transplant 6: 1639-1645, 2006.

13. Trachtman H, Christen E, Cnaan A, Patrick J, Mai V, Mishra J, Jain A, Bullington N, Devarajan P and Investigators of the HUS-SYNSORB Pk Multicenter Clinical Trial: Urinary neutrophil gelatinase-associated lipocalcin in D+HUS: A novel marker of renal injury. Pediatr Nephrol 21: 989-994, 2006.

14. Hirsch R, Dent C, Pfriem H, et al: NGAL is an early predictive biomarker of contrast-induced nephropathy in children. Pediatr Nephrol 22: 2089-2095, 2007.

15. Du Y, Hou L, Guo J, Sun T, Wang X and Wu Y: Renal neutrophil gelatinase-associated lipocalin and kidney injury molecule-1 expression in children with acute kidney injury and Henoch-Schönlein purpura nephritis. Exp Ther Med 7: $1130-1134,2014$

16. Cho E, Yang HN, Jo SK, Cho WY and Kim HK: The role of urinary liver-type fatty acid-binding protein in critically il patients. J Korean Med Sci 28: 100-105, 2013.

17. de Geus HR, Bakker J, Lesaffre EM and le Noble JL: Neutrophil gelatinase-associated lipocalin at ICU admission predicts for acute kidney injury in adult patients. Am J Respir Crit Care Med 183: 907-914, 2011.

18. Christie B: Doctors revise declaration of Helsinki. BMJ 321: 913 , 2000.

19. Costa e Silva VT, de Castro I, Liaño F, Muriel A Rodríguez-Palomares JR and Yu L: Sequential evaluation of prognostic models in the early diagnosis of acute kidney injury in the intensive care unit. Kidney Int 75: 982-986, 2009.

20. Palsson R and Niles JL: Regional citrate anticoagulation in continuous venovenous hemofiltration in critically ill patients with a high risk of bleeding. Kidney Int 55: 1991-1997, 1999.

21. Haase M and Haase-Fielitz A: Neutrophil gelatinase-associated lipocalin for acute kidney injury - the renal troponin. J Lab Med 165: 28-32, 2010

22. Feltes CM, Van Eyk J and Rabb H: Distant-organ changes after acute kidney injury. Nephron, Physiol 109: 80-84, 2008.

23. Lanore JJ, Brunet F, Pochard F, et al: Hemodialysis for acute renal failure in patients with hematologic malignancies. Crit Care Med 19: 346-351, 1991

24. Benoit DD, Hoste EA, Depuydt PO, Offner FC, Lameire NH, Vandewoude KH, Dhondt AW, Noens LA and Decruyenaere JM: Outcome in critically ill medical patients treated with renal replacement therapy for acute renal failure: Comparison between patients with and those without haematological malignancies. Nephrol Dial Transplant 20: 552-558, 2005

25. Azoulay E, Recher C, Alberti C, Soufir L, Leleu G, Le Gall JR, Fermand JP and Schlemmer B: Changing use of intensive care for hematological patients: The example of multiple myeloma. Intensive Care Med 25: 1395-1401, 1999.

26. Azoulay E, Moreau D, Alberti C, Leleu G, Adrie C, Barboteu M, Cottu P, Levy V, Le Gall JR and Schlemmer B: Predictors of short-term mortality in critically ill patients with solid malignancies. Intensive Care Med 26: 1817-1823, 2000.

27. Darmon M, Thiery G, Ciroldi M, de Miranda S, Galicier L, Raffoux E, Le Gall JR, Schlemmer B and Azoulay E: Intensive care in patients with newly diagnosed malignancies and a need for cancer chemotherapy. Crit Care Med 33: 2488-2493, 2005.

28. Bagshaw SM, Laupland KB, Doig CJ, Mortis G, Fick GH, Mucenski M, Godinez-Luna T, Svenson LW and Rosenal T: Prognosis for long-term survival and renal recovery in critically ill patients with severe acute renal failure: A population-based study. Crit Care 9: R700-R709, 2005.
29. Mishra J, Mori K, Ma Q, Kelly C, Barasch J and Devarajan P: Neutrophil gelatinase-associated lipocalin: A novel early urinary biomarker for cisplatin nephrotoxicity. Am J Nephrol 24: 307-315, 2004

30. Mehta RL, Kellum JA, Shah SV, Molitoris BA, Ronco C, Warnock DG, Levin A and Acute Kidney Injury Network: Acute Kidney Injury Network: Report of an initiative to improve outcomes in acute kidney injury. Crit Care 11: R31, 2007.

31. Waikar SS and Bonventre JV: Biomarkers for the diagnosis of acute kidney injury. Nephron Clin Pract 109: c192-c197, 2008.

32. Haase M, Bellomo R, Devarajan P, Schlattmann P, Haase-Fielitz A and NGAL Meta-analysis Investigator Group: Accuracy of neutrophil gelatinase-associated lipocalin (NGAL) in diagnosis and prognosis in acute kidney injury: A systematic review and meta-analysis. Am J Kidney Dis 54: 1012-1024, 2009.

33. Haase-Fielitz A, Haase M and Devarajan P: Neutrophil gelatinase-associated lipocalin as a biomarker of acute kidney injury: A critical evaluation of current status. Ann Clin Biochem 51: 335-351, 2014.

34. Mishra J, Dent C, Tarabishi R, et al: Neutrophil gelatinase-associated lipocalin (NGAL) as a biomarker for acute renal injury after cardiac surgery. Lancet 365: 1231-1238, 2005.

35. Parikh CR, Devarajan P, Zappitelli M, et al: Postoperative biomarkers predict acute kidney injury and poor outcomes after pediatric cardiac surgery. J Am Soc Nephrol 22: 1737-1747, 2011.

36. Zappitelli M, Washburn KK, Arikan AA, Loftis L, Ma Q, Devarajan P, Parikh CR and Goldstein SL: Urine NGAL is an early marker of acute kidney injury in critically ill children. Crit Care 11: R84, 2007.

37. Du Y, Zappitelli M, Mian A, Bennett M, Ma Q, Devarajan P, Mehta R and Goldstein SL: Urinary biomarkers to detect acute kidney injury in the pediatric emergency center. Pediatr Nephrol 26: 267-274, 2011.

38. Wheeler DS, Devarajan P, Ma Q, Harmon K, Monaco M, Cvijanovich N and Wong HR: Serum neutrophil gelatinase-associated lipocalin (NGAL) as a marker of acute kidney injury in critically ill children with septic shock. Crit Care Med 36: 1297-1303, 2008

39. Martensson J, Bell M, Oldner A, Xu S, Venge P and Martling CR: Neutrophil gelatinase-associated lipocalin in adult septic patients with and without acute kidney injury. Intensiv Care Med 36 $1333-1340,2010$

40. Nickolas TL, Schmidt-Ott KM, Canetta P, et al: Diagnostic and prognostic stratification in the emergency department using urinary biomarkers of nephron damage: A multicenter prospective cohort study. J Am Coll Cardiol 59: 246-255, 2012.

41. Rahimzadeh N, Otukesh H, Hoseini R, Sorkhi H, Otukesh M, Hoseini S and Torkzaban M: Are serum and urine neutrophil gelatinase-associated lipocalin predictive of renal graft function in short term. Pediatr Transplant 16: 796-802, 2012.

42. Royakkers AA, Bouman CS, Stassen PM, Korevaar JC, Binnekade JM, van de Hoek W, Kuiper MA, Spronk PE and Schultz MJ: Systemic and urinary neutrophil gelatinase-associated lipocalins are poor predictors of acute kidney injury in unselected critically ill patients. Crit Care Res Pract 2012: 712695, 2012.

43. Endre ZH, Pickering JW, Walker RJ, et al: Improved performance of urinary biomarkers of acute kidney injury in the critically ill by stratification for injury duration and baseline renal function. Kidney Int 79: 1119-1130, 2011.

44. Metzger J, Kirsch T, Schiffer E, Ulger P, Mentes E, Brand K, Weissinger EM, Haubitz M, Mischak $\mathrm{H}$ and Herget-Rosenthal S: Urinary excretion of twenty peptides forms an early and accurate diagnostic pattern of acute kidney injury. Kidney Int 78: 1252-1262, 2010

45. Daw NC, Gregornik D, Rodman J, Marina N, Wu J, Kun LE, Jenkins JJ, McPherson V, Wilimas J and Jones DP: Renal function after ifosfamide, carboplatin and etoposide (ICE) chemotherapy, nephrectomy and radiotherapy in children with Wilms tumour. Eur J Cancer 45: 99-106, 2009

46. Bailey S, Roberts A, Brock C, Price L, Craft AW, Kilkarni R, Lee RE, Skillen AW and Skinner R: Nephrotoxicity in survivors of Wilms' tumours in the North of England. Br J Cancer 87: 1092-1098, 2002. 